\title{
Off-Line Feed Rate Scheduling Based on a Mechanistic Cutting Force on Discrete Segments during End Milling
}

\author{
M. N. Islam ${ }^{1, a}$, A. Pramanik ${ }^{1, b}$, A. K. Basak ${ }^{2, c}$ \\ ${ }^{1}$ Department of Mechanical Engineering, Curtin University, Bentley, WA, Australia \\ ${ }^{2}$ Adelaide Microscopy, University of Adelaide, Adelaide, SA, Australia \\ am.n.islam@curtin.edu.au, balokesh.pramanik@curtin.edu.au canimesh.basak@ adelaide.edu.au,
}

Keywords: off-line, feed rate scheduling, discrete segments, cutting force, end milling

\begin{abstract}
This paper describes the development of an off-line feed rate scheduling technique based on a mechanistic cutting force model. The proposed technique was developed for an end milling operation. The surface area of the workpiece was divided into a number of segments, and the resultant cutting force at each discrete segment was determined using One Path Analysis software. The calculated resultant cutting force was applied to the feed rate scheduling. Experimental results clearly showed that the implementation of feed rate scheduling reduces machining time considerably and that as the number of segments increases, the effectiveness of the feed rate scheduling increases.
\end{abstract}

\section{Introduction}

Machining processes shape parts by removing unwanted materials [1]. In the machining process, one must follow given quality specifications, such as surface finish, accuracy, and surface integrity [2]. The manufacturing standard has changed from mass production to mass customization, and many manufacturing industries require new technologies to increase production rate, reduce production costs, and adapt this transition. Existing CAM technology does not consider important physical properties, such as cutting force and machined surfaces [3]. Thus, optimal selection of cutting conditions still requires the expertise and experience of a skilled machinist or the information from a machining data handbook.

There have been many investigations on feed rate scheduling, most of which are based on volumetric analysis by using the material removal rate [3, 4]. Ip et al. [5] and Wang et al. [6] developed algorithms for adjusting the feed rate by optimizing the material removal rate to increase machining efficiency. Li [7] integrated CAD/CAM and related the average power with the material removal rate to optimize the off-line feed rate. Lee et al. [8] introduced intelligent off-line feed rate scheduling where the highest value of the cutting forces was amended to a constant value by dividing the original blocks of NC codes into smaller ones with the optimized feed rate values. A feed rate scheduling method guided by splines for machining along curvilinear paths with real-time application of chord errors and acceleration/deceleration constraints was developed by Sun [9]. Merdol [10] calculated optimum feed rate levels to maximize the material removal rate by a solid modeller approach in order to simulate the cutting forces in three-axis machining of dies and molds. Several investigators have studied off-line feed rate scheduling based on mechanistic cutting force models [3, 11]. Lim et al. [12], for example, suggested a cutting path adaptive feed rate strategy based on force and dimensional accuracy. Ko et al. [13, 14] adjusted acceleration/deceleration time of the controller to schedule an off-line feed rate based on the mechanistic cutting force model for flat end milling. Chen et al. [15] scheduled a feed rate for high-speed ball-end milling for cases where the scallop height of the feed interval limited high efficiency machining.

The mechanistic cutting force model is considered to accurately predict cutting forces [16]. There have been numerous investigations of off-line feed rate scheduling based on mechanistic force models. However, documented feed rate scheduling is based on complex equations with little or no practical application. At this stage, an off-line feed rate scheduling method that is simple to use, acceptable to the industrial community, and highly productive is needed. In this study, off-line feed 
rate scheduling has been developed based on the mechanistic cutting force on discrete segments during end milling. We used One Path Analysis software and NC code modifications. The end milling process has been extensively analyzed to improve the machining operation economically. The experimental results show that the machining time is reduced by $55.1 \%$ at a scheduled feed rate.

\section{Feed rate scheduling strategies}

Feed rate scheduling based on the material removal rate is a commonly used strategy. Feed rate is proportional to either the average or instantaneous volumetric removal rate. Commercial software packages, such as Vericut's feed rate optimization module Optipath and Mastercam's Hifeed, work on volumetric analysis [16]. Theoretically, efficient high-performance machining is achieved by maximizing the material removal rate. However, the predictions from this strategy show and emphasize that the scheduled feed rate value causes greater forces, which can damage the cutting tool and CNC machine and cause a higher machined-surface error [16]. Off-line feed rate scheduling regulates the constant feed rate according to the reference cutting force exerted on the cutting tool. In order to increase the resultant force to the desired reference cutting force, a simple linear relation is found between the feed rate and the reference limiting cutting force [16]. The relationship between the feed rate and force can be established for the selection of optimum feed rate values - the faster the feed rate, the greater the cutting forces. However, increasing cutting forces causes various problems, such as machining chatter, tool wear, and tool breakage. Thus, it is important to determine the characteristics of the end mill tool, such as the tool material, number of flutes, rake and helix angle, diameter, length, modulus of elasticity, and the maximum forces allowable, in order to achieve an effective feed rate scheduling. In the mechanistic cutting force model, machining parameters, such as rotational speed of cutter, type, and characteristic of cutter, and the material and geometry of the workpiece, are set as constant during feed rate scheduling.

\section{Model development}

Feed rate scheduling plays a major role in optimizing the machining operation by fully utilizing the cutter. It drastically reduces machine time and increases productivity. Feed rate scheduling requires accurate predictions of the cutting force waveforms and magnitudes. One Path Analysis software was used to determine the $\mathrm{x}, \mathrm{y}, \mathrm{z}$ forces with respect to the end mill cutter rotation angle and cutting coefficients. The software was developed by Professor Dong Woo Cho, in the Department of Mechanical Engineering at Pohang University of Science \& Technology, South Korea. A reference cutting force is required to implement feed rate scheduling. Lee et al. [17] developed a reference cutting force model using FEM analysis and showed that a cutting force of less than $704 \mathrm{~N}$ provides a safe cutting process. Considering our case, a reduction factor of 0.75 was implied, and we set the reference cutting force for our test to 525 N. One Path Analysis software was used to calculate the cutting forces acting on the end mill cutter. It is important to set all the cutting parameters as constant before determining the cutting forces and effectively implementing the feed rate scheduling.

The geometry of the set workpiece is shown in Fig. 1a; the material of the workpiece was Aluminum 6061. The four-fluted HSS straight shank end mill (IZAR Cutting Tools) used in this experiment had the following parameters: diameter of $16 \mathrm{~mm}$, nominal helix angle of $30^{\circ}$, rake angle of $10^{\circ}$, modulus of elasticity of $217,000 \mathrm{~N} / \mathrm{mm}^{2}$, and cutter length of $32 \mathrm{~mm}$ (Fig. 1b). The spindle speed was set at $1500 \mathrm{rpm}$ throughout the test. The workpiece to be machined was divided into 20 (Fig. 2a), 30 (Fig. 2b), 40, 60, and 120 segments. The radial depth of cut was set constant at $2 \mathrm{~mm}$, and the axial depth of cut for each segment was from 10.167 to $30 \mathrm{~mm}$ depending on the number of divisions. The resultant cutting force acting at each discrete element for 20, 30, 40, 60, and 120 segments was determined using One Path Analysis software. The workpiece segments were subdivided because of the variations in the axial cutting depth. A higher number of segments led to a more accurate prediction of the resultant cutting forces at each discrete segment. Before determining the cutting force, a constant feed rate was set as a reference since One Path Analysis software requires 
a feed rate value to calculate the cutting forces. The reference feed rate was set at $466 \mathrm{~mm} / \mathrm{min}$ to deploy the maximum capacity of the cutter. Fig. 3 illustrates the method to determine the reference feed rate. The resultant cutting forces were then determined based on the reference feed rate at each individual segment of the $20,30,40,60$, and 120 segments. The technique used to determine the resultant force for 20 segments is described below.

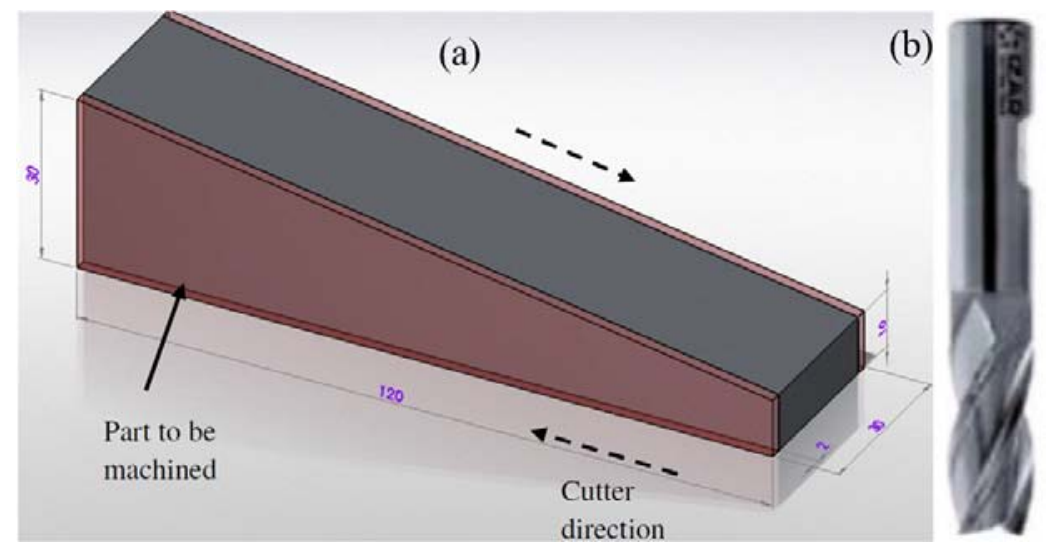

Fig. 1 (a) Geometry of Aluminum 6061 (b) 16 mm HSS straight shank end mill

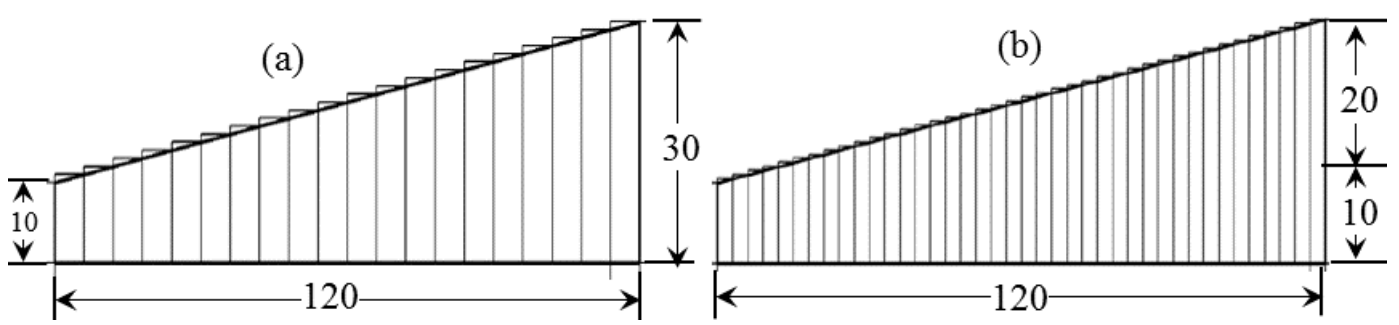

Fig. 2 Parts to be machined were divided into (a) 20 segments (b) 30 segments

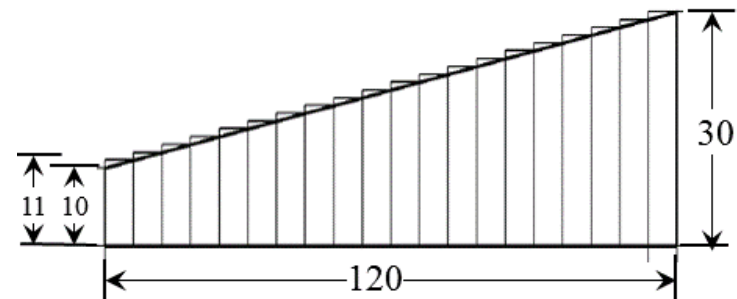

(a)

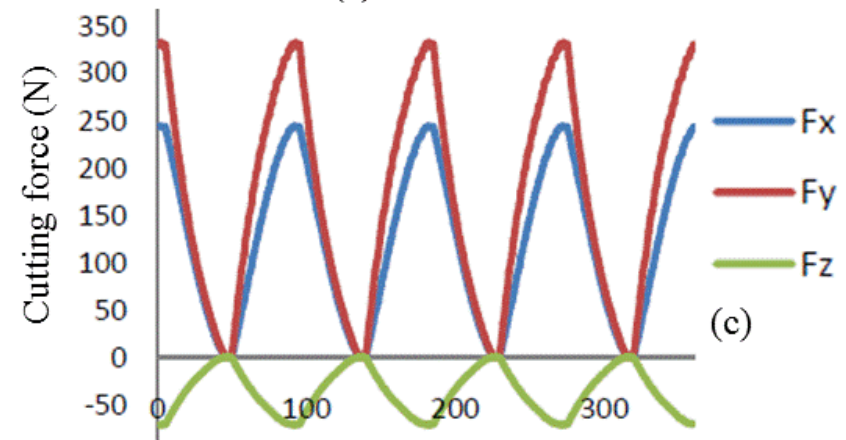

Cutter rotation angle $\left(^{\circ}\right)$ (b)

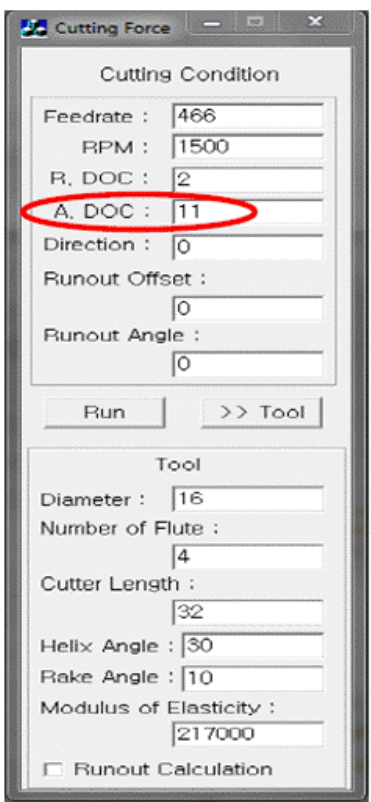

Fig. 3 Resultant force for 20 segments (a) height of segment, (b) input parameters in One Path Analysis software, and (c) cutting forces vs. cutter rotation angle 
The height of the first segment was measured. Cutting conditions were set in One Path Analysis software. The feed rate was $466 \mathrm{~mm} / \mathrm{min}$, and the radial depth of cut was $2 \mathrm{~mm}$. The height of the first segment, which is also the axial depth of cut of the cutter, was $11 \mathrm{~mm}$. The cutting forces at each rotation angle of the cutter were calculated by the software. Fig. 3c shows the cutting forces at each angle. The resultant force acting on the cutter was determined (192.67 N). The same method, with only a change in the axial depth of cut, was used to calculate the remaining resultant cutting forces for the various segments (Fig. 4). The forces show a linear relationship with the length of segments and advancement in the $x$ direction. After determining all the cutting forces, feed rate schedules were implemented by increasing the cutting forces.

Fig. 5 illustrates the feed rate schedules in a polynomial relationship for 20 and 120 segments. A similar method was applied for 30, 40, 60, and 120 segments. The arrow in Fig. 6 indicates that the constant feed rate of $466 \mathrm{~mm} / \mathrm{min}$ increased as the axial depth of cut to be machined decreased in order to maximize efficiency. A new feed rate value was assumed as long the resultant cutting force exceeded $525 \mathrm{~N}$. Thus, a resultant cutting force of $540.30 \mathrm{~N}$ at a $1560-\mathrm{mm} / \mathrm{min}$ feed rate was calculated. The feed rate value at a cutting force of $525 \mathrm{~N}$ can be determined using the interpolation method, and this gave a new feed rate value of $1511.84 \mathrm{~mm} / \mathrm{min}$.

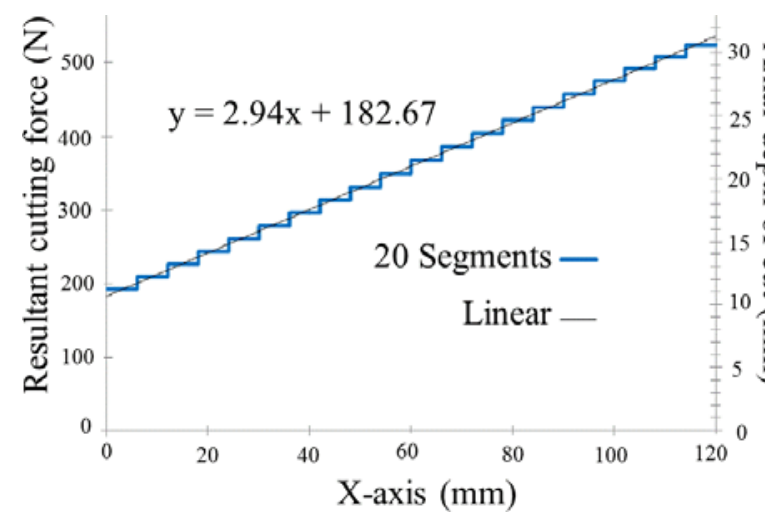

(a)

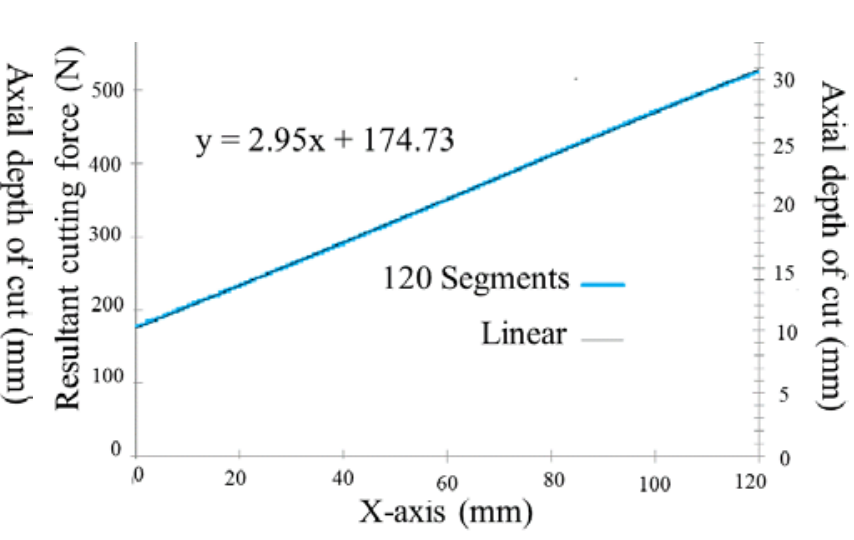

(b)

Fig. 4 Resultant force as the axial depth of cut varies along the $x$-axis.

(a) 20 segments; (b) 120 segments

\section{Off-line feed rate scheduling (NC codes)}

Off-line NC codes were developed based on the feed rate scheduling as determined above. CNC simulation software (Alphacam Ultimate Mill) was used to verify the validity of the developed NC codes. Table 1 lists the application of the off-line feed rate schedules for 20, 30, and 60 segments. Similarly, NC codes were generated for 40 and 120 segments (data not presented here due to lack of space). 


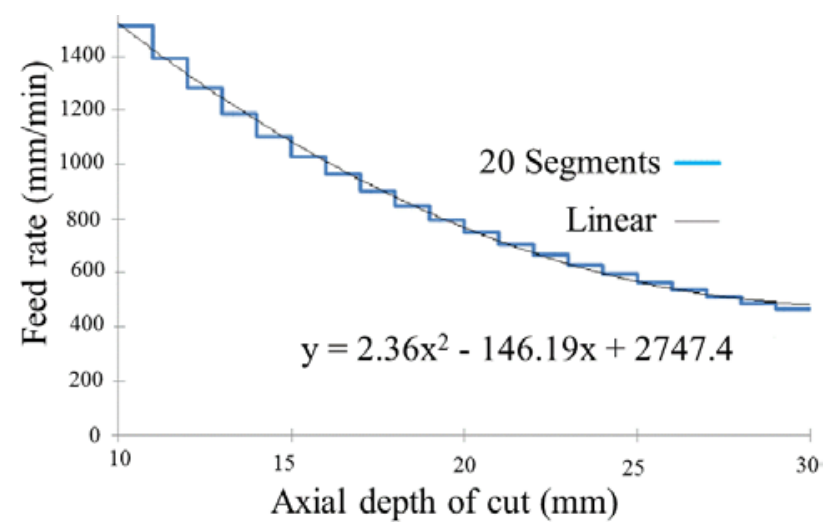

(a)

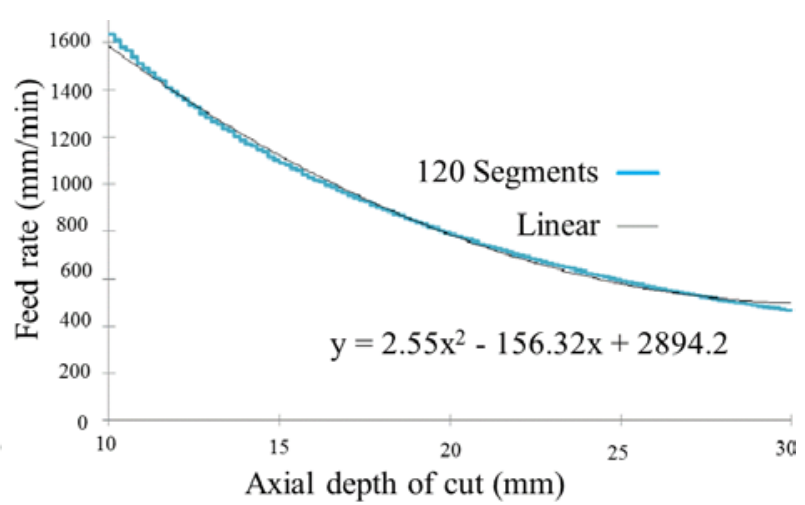

(b)

Fig. 5 Feed rate schedules for 20 and 120 segments as the axial depth of cut increases along the $x$-axis

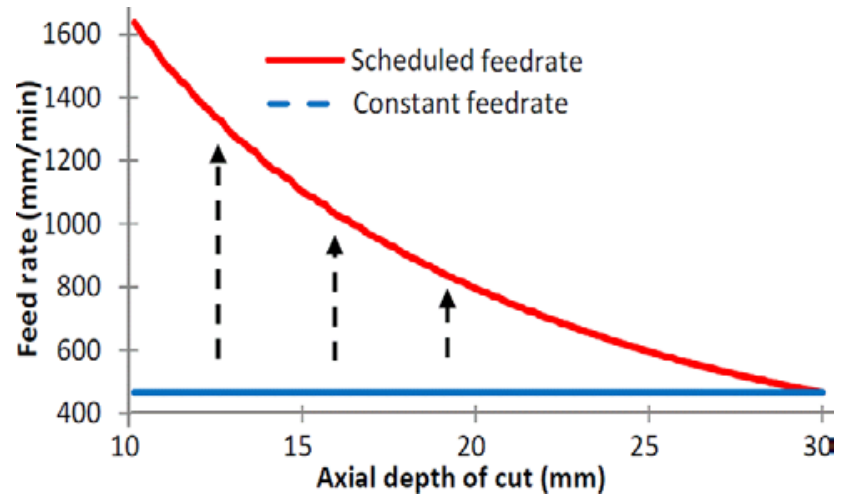

Fig. 6 Difference between before and after feed rate scheduling

Table 1 Off-line feed rate scheduling NC codes for 20 and 60 segments

\begin{tabular}{|c|c|c|}
\hline \multicolumn{3}{|c|}{ Constant feed rate } \\
\hline \multirow{3}{*}{$\begin{array}{l}\text { START } \\
\% \\
\text { N10 G90 G40 G49; } \\
\text { N20 M06 T09; }\end{array}$} & & \multirow{3}{*}{$\begin{array}{l}\text { N40 M03 S1500; } \\
\text { N45 G04 P1000; } \\
\text { N50 G91 G01 X150.00 F466; } \\
. . .\end{array}$} \\
\hline & N45 G( & \\
\hline & N50 Gs & \\
\hline \multicolumn{3}{|c|}{ Feed rate schedule when the part was divided into 20 segments } \\
\hline N80 G01 X-16.00 F1512; & \multirow{7}{*}{$\begin{array}{l}\text { N150 G01 X-6.00 F902; } \\
\text { N160 G01 X-6.00 F846; } \\
\text { N170 G01 X-6.00 F795; } \\
\text { N180 G01 X-6.00 F748; } \\
\text { N190 G01 X-6.00 F705; } \\
\text { N200 G01 X-6.00 F665; } \\
\text { N210 G01 X-6.00 F629; }\end{array}$} & N220 G01 X-6.00 F595; \\
\hline N90 G01 X-6.00 F1392; & & N230 G01 X-6.00 F565; \\
\hline N100 G01 X-6.00 F1283; & & N240 G01 X-6.00 F537; \\
\hline N110 G01 X-6.00 F1186; & & N250 G01 X-6.00 F511; \\
\hline N120 G01 X-6.00 F1102; & & N260 G01 X-6.00 F488; \\
\hline N130 G01 X-6.00 F1028; & & N270 G01 X-26.00 F466; \\
\hline N140 G01 X-6.00 F963; & & \\
\hline \multicolumn{3}{|c|}{ Feed rate schedule when the part was divided into 60 segments } \\
\hline N50 G91 G01 X12.00 F466; & N260 G01 X2.00 F654; & N460 G01 X2.00 F990; \\
\hline N70 G01 X2.00 F474; & N270 G01 X2.00 F665; & N470 G01 X2.00 F1010; \\
\hline N80 G01 X2.00 F481; & N280 G01 X2.00 F680; & N480 G01 X2.00 F1028; \\
\hline N90 G01 X2.00 F488; & N290 G01 X2.00 F693; & N490 G01 X2.00 F1060; \\
\hline N100 G01 X2.00 F497; & N300 G01 X2.00 F705; & N500 G01 X2.00 F1082; \\
\hline N110 G01 X2.00 F504; & N310 G01 X2.00 F722; & N510 G01 X2.00 F1102; \\
\hline N120 G01 X2.00 F511; & N320 G01 X2.00 F735; & N520 G01 X2.00 F1139; \\
\hline N130 G01 X2.00 F521; & N330 G01 X2.00 F748; & N530 G01 X2.00 F1165; \\
\hline
\end{tabular}




\begin{tabular}{|l|l|l|}
\hline N140 G01 X2.00 F528; & N340 G01 X2.00 F766; & N540 G01 X2.00 F1186; \\
N150 G01 X2.00 F537; & N350 G01 X2.00 F781; & N550 G01 X2.00 F1227; \\
N160 G01 X2.00 F547; & N360 G01 X2.00 F795; & N560 G01 X2.00 F1255; \\
N170 G01 X2.00 F556; & N370 G01 X2.00 F815; & N570 G01 X2.00 F1283; \\
N180 G01 X2.00 F565; & N380 G01 X2.00 F830; & N580 G01 X2.00 F1326; \\
N190 G01 X2.00 F576; & N390 G01 X2.00 F846; & N590 G01 X2.00 F1358; \\
N200 G01 X2.00 F585; & N400 G01 X2.00 F868; & N600 G01 X2.00 F1392; \\
N210 G01 X2.00 F595; & N410 G01 X2.00 F885; & N610 G01 X2.00 F1438; \\
N220 G01 X2.00 F608; & N420 G01 X2.00 F902; & N620 G01 X2.00 F1475; \\
N230 G01 X2.00 F618; & N430 G01 X2.00 F926; & N630 G01 X2.00 F1512; \\
N240 G01 X2.00 F629; & N440 G01 X2.00 F944; & N640 G01 X2.00 F1568; \\
N250 G01 X2.00 F643; & N450 G01 X2.00 F963; & N650 G01 X22.00 F1610; \\
\hline
\end{tabular}

\section{Model validations}

In order to test the effectiveness of the off-line feed rate scheduling, experimental validation tests were performed on a Leadwell V-30 Vertical Machining Center, using an Al 6061 workpiece and an HSS straight shank end mill. The developed off-line feed rate scheduling NC codes were transferred from a PC into a FANUC Series 21-M to generate the desired cutting tool path. Table 1 illustrates the application of the off-line feed rate scheduling in the NC codes and the schematic tool path. Once the cutter started cutting operations, the time was recorded for each part machined. The time taken to machine a part at a constant feed rate was set as the time reference. Table 2 and Fig. 7 show the comparison in machining time between before (constant feed rate) and after (feed rate schedule values between 20 and 120 segments) feed rate scheduling.

Table 2 Comparison of machining time before and after applying feed rate scheduling

\begin{tabular}{lcc}
\hline & Machine time (s) & Reduction (\%) \\
Without feed rate scheduling & 20.5 & \\
Feed rate scheduling (20 segments) & 15.8 & 22.9 \\
Feed rate scheduling (30 segments) & 14.3 & 30.2 \\
Feed rate scheduling (40 segments) & 10.7 & 47.8 \\
Feed rate scheduling (60 segments) & 10.0 & 51.2 \\
Feed rate scheduling (120 segments) & 9.2 & 55.1 \\
\hline
\end{tabular}

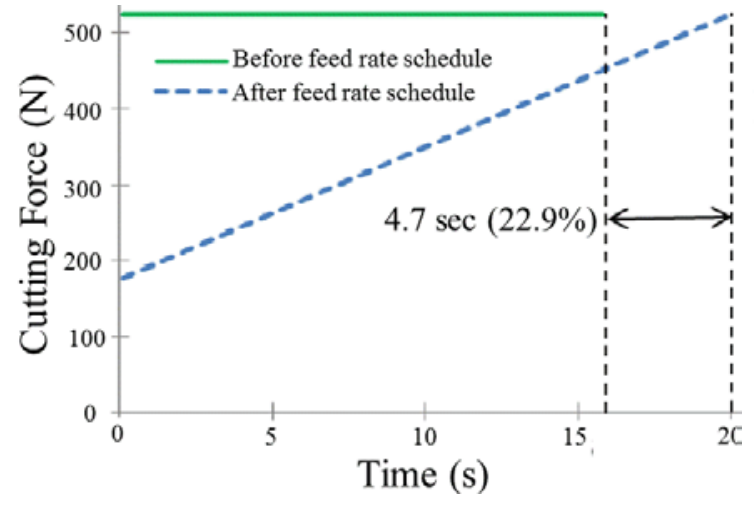

(a)

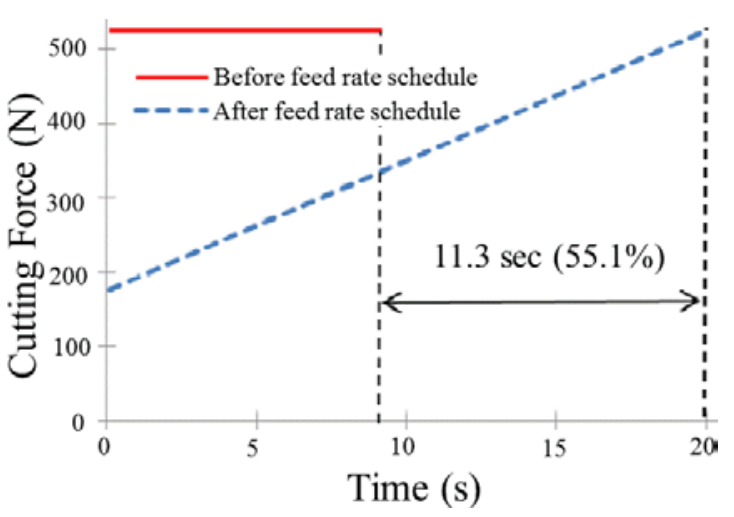

(b)

Fig. 7 Machine time before and after implementing feed rate scheduling.

(a) 20 segments; (b) 120 segments 


\section{Summary}

Many manufacturing companies are struggling to fulfill the high demand for increased productivity while sustaining the quality of manufactured components in a short time and in an economical way. Thus, the application of feed rate scheduling plays a major role in optimizing the machining operations by reducing machining time. In this paper, off-line feed rate schedules were developed based on a mechanistic cutting force model and the effectiveness was tested. We found that with a higher number of segments, the predicted cutting force was more accurate. Experimental results showed that the scheduled feed rate based on 120 segments consumed the least machine time (9.2 sec). Machining time was reduced by 55.1\%. Thus, the application of feed rate scheduling is an ideal method for machining optimization.

\section{Acknowledgement}

The authors acknowledge the contribution of Mr. Marko K. S. Chua, a final year student at the Department of Mechanical Engineering, Curtin University, Australia.

\section{References}

[1] A. Pramanik, K.S. Neo, M. Rahman, et al., J of Mat. Proce. Tech., 208(1)(2008) p.400

[2] A. Pramanik, K.S. Neo, M. Rahman, et al., Int. J. of Adv. Manuf. Tech. 43 (7)(2009) p.681

[3] H. Erdim, I. Lazoglu, B. Ozturk, Int. J of Machine Tools and Manuf., 46(7)(2006) p.747

[4] K.S.E. Layegh, H. Erdim, I. Lazoglu, Procedia CIRP, 1 (2012) p.96

[5] R.W. Ip, H.C. Lau, F.T. Chan, J of Mat. Processing Technology, 138(1)(2003) p.579

[6] W. P. Wang, Journal of Manufacturing Systems, 7(1)(1988) p.57

[7] Z.Z. Li, M. Zheng, L. Zheng, Z.J. Wu, D.C. Liu, J of Mat. Proce. Tech. 138(1)(2003) p.513

[8] H.U. Lee, D.W. Cho, Int. J. of Adv. Manuf. Tech., 22(11-12)(2003) p.873

[9] Y. Sun, Z. Jia, F. Ren, D. Guo, Int. J. of Advan. Manuf. Tech., 36(1-2)(2008) p.60

[10] S.D. Merdol, Y. Altintas,. Int. J of Machine Tools and Manuf., 48(10)(2008) p.1063

[11] K.S.E. Layegh, H. Erdim, I. Lazoglu, Procedia CIRP, 1 (2012) p. 96

[12] E.E.M. Lim, C.H. Menq, Int. J. of Machine Tools and Manuf. 37 (1)(1997) p.61

[13] J.H. Ko, W.S. Yun, D.W. Cho, Computer-Aided Design, 35(4)(2003) p.383

[14] J.H. Ko, D.W. Cho, Int. J.of Machine Tools and Manuf., 44(10)(2004) p.1047

[15] J.S. Chen, Y.K. Huang, M.S. Chen, Int. J of Machine Tools and Manuf., 45(9)(2005) p.1076

[16] A. Pramanik, L.C. Zhang, et al., Int. J of Machine Tools and Manuf., 46(14)(2006) p.1795

[17] H.U. Lee, D.W. Cho, Int. J of Machine Tools and Manuf., 47(1)(2007) p.158 